\title{
Avaliação do Seguimento Clínico de Citopatologia Oncótica em Mulheres na Atenção Primária à Saúde
}

\section{Evaluation of Clinical Follow of Cytopathology Oncotic in Women in Primary Health Care}

\author{
Cláudia Janiele Batista Fonsêca ${ }^{1}$ \\ Tainara Lôrena dos Santos Ferreira ${ }^{2}$ \\ Daísy Vieira de Araújo ${ }^{3}$ \\ Káthya Daniella Figueiredo Melo ${ }^{4}$ \\ Fábia Barbosa de Andrade ${ }^{5}$
}

\section{RESUMO}

Objetivo: Este estudo avaliou o seguimento clínico e terapêutico da citopatologia oncótica em mulheres na Atenção Primária à Saúde (APS). Métodos: Trata-se de um estudo exploratóriodescritivo, de abordagem quantitativa, realizado na Cidade de Santa Cruz/RN, Brasil, o qual se utilizou de uma amostra de 150 mulheres. A pesquisa foi aprovada pelo Comitê de Ética em Pesquisa da Universidade Federal do Rio Grande do Norte, sob número de parecer 313.430. Resultados: Observou-se que, no tocante ao resultado do exame preventivo, $66 \%$ da amostra referiu ter apresentado Cândida albicans, seguido por $28,7 \%$ que apresentaram positividade de Gardnerella vaginallis, e que $4,7 \%$ tiveram resultados positivos para HPV e NIC I. Conclusões: Observou-se a continuidade da assistência para mulheres que apresentaram NIC I e HPV, no entanto, espera-se que os profissionais de saúde estimulem o acompanhamento da mulher na rede básica de saúde e, consequentemente, fortaleçam o vínculo entre o usuário-serviço, de modo que favoreça a assistência.

DESCRITORES: Saúde da Mulher, Teste de Papanicolaou, Neoplasias do Colo do Útero, Enfermagem.

\begin{abstract}
Objective: This study aims to evaluate the Clinical and therapeutic follow-up of oncotic cytopathology in women in Primary Health Care (PHC). Methods: This was an exploratory descriptive study with a quantitative approach, in the city of Santa Cruz / RN, Brazil, which used a sample of 150 women. The study was approved by the Ethics Committee in Research of the Federal University of Rio Grande do Norte, under number 313 430. Results: It was observed that, in relation to the result of the screening test, $66 \%$ of the sample reported having presented Candida albicans, followed by $28.7 \%$ who were positive for Gardnerella vaginallis, and $4.7 \%$ were positive for HPV and $\mathrm{CIN} \mathrm{I.} \mathrm{Conclusions:} \mathrm{it} \mathrm{was} \mathrm{observed} \mathrm{the} \mathrm{continuity} \mathrm{of} \mathrm{care} \mathrm{for}$ women with CIN I and HPV, however, it is expected that health professionals encourage the monitoring of women in primary health care network and thus strengthen the bond between the user-service, so that it can favor assistance.
\end{abstract}

DESCRIPTORS: Women's Health, Papanicolaou Test, Uterine Cervical Neoplasms, Nursing.

1 - Estudante do Curso de Graduação em Enfermagem da Universidade Federal do Rio Grande do Norte - UFRN, Santa Cruz, Rio Grande do Norte.

2 - Estudante do Curso de Graduação em Enfermagem da Universidade Federal do Rio Grande do Norte - UFRN. Bolsista de Iniciação Científica pelo CNPq. Santa Cruz, Rio Grande do Norte.

3 - Gerente da Atenção Básica da Prefeitura Municipal de Cuité, Cuité, Paraíba.

4 - Professora do Curso de Graduação em Enfermagem da Universidade Federal do Rio Grande do Norte - UFRN, Santa Cruz, Rio Grande do Norte.

5 - Professora da Universidade Federal do Rio Grande do Norte - UFRN, Natal, Rio Grande do Norte. 
A s doenças oncológicas são temidas por todo o mundo, pois causam muito sofrimento às pessoas que são acometidas por esse tipo de doença. Os cânceres que mais afetam as mulheres e são mais temidos por elas são o de mama e o de colo do útero, porque ambos atingem a mulher no seu ponto fraco, mexem com sua feminilidade.

O câncer do colo do útero (CCU) apresentase como o segundo tipo de câncer mais comum em mulheres e o maior causador de morte em mulheres jovens, principalmente em países de terceiro mundo. ${ }^{1}$ Com o inicio da reforma sanitária no final de 1980 e a criação do Sistema Único de Saúde (SUS), o perfil do controle do câncer do colo do útero no Brasil começou a mudar. A partir de 1995, com as demandas do movimento feminista e a participação do Brasil na VI Conferencia Internacional da mulher, organizou-se uma grande campanha de rastreamento de câncer do colo do útero por meio da qual se constituiu o projeto Viva Mulher, que foi implantado pelo Instituto Nacional do Câncer (INCA), visando desenvolver as bases de estruturação de um programa de rastreamento do câncer do colo do útero de forma que abrangesse todo o país. ${ }^{2}$

O Brasil é um país que tem uma população, em sua maioria, constituída por mulheres (50,77\%), as quais são as principais usuárias do Sistema Único de Saúde. Mulheres que buscam os serviços de saúde por várias particularidades, tanto para seu próprio atendimento, como também para o acompanhando de familiares e amigos. ${ }^{3}$ As estimativas de incidência de câncer do colo do útero para o Brasil em 2012 foram de 17.540 novos casos, com estimativas de 5.050 para a região Nordeste e 230 novos casos, por 100 mil habitantes, para o Estado do Rio Grande do Norte. ${ }^{4}$

O CCU é um câncer com mortalidade elevada e, mesmo com as campanhas e programas governamentais de prevenção, ainda continua sendo um problema de saúde pública no Brasil, embora já apresente conhecimentos técnicos de prevenção suficientes para fornecer um dos mais altos potenciais de cura. ${ }^{5}$

Um dos atributos propostos pelo Sistema Único de Saúde é a integralidade na assistência à saúde de qualquer indivíduo, com o objetivo de atender às necessidades de saúde e atuar na educação, prevenção e manutenção da saúde. Então, partindo desse pressuposto, segundo os autores $^{6}$, a integralidade da atenção à saúde das mulheres também pressupõe que estas, em algum momento de suas vidas, buscam os serviços de saúde, seja para suas próprias necessidades, seja para as de familiares. Nesses momentos, essas mulheres podem ser orientadas quanto à importância dos cuidados necessários à prevenção contra a infecção pelo vírus do HPV, ou para a forma de detecção precoce.

A Atenção Primária à Saúde é a porta de entrada para a assistência com foco na prevenção e promoção e, assim, responsável pelas medidas preventivas, educativas e de tratamento das doenças, antes do seu agravamento, haja vista que quando se tem uma boa atenção à saúde evitam-se complicações e favorece a diminuição das lotações nos outros níveis de atenção à saúde.

O primeiro nível de atenção, chamado Atenção Primária à Saúde (APS), no Brasil também intitulado de Atenção Básica, tem papel importante e crucial no que diz respeito à detecção precoce do câncer uterino, bem como de outras doenças. Pois, mesmo que os programas do câncer envolvam todos os níveis de atenção no seu cuidado, este cuidado em nível básico desenvolve as ações preventivas e de detecção precoce, uma vez que a APS é o primeiro contato dos indivíduos com os serviços de saúde. A atuação no nível primário pode evitar o aparecimento da doença ao utilizar intervenções em seus fatores de risco, como o estímulo ao sexo seguro, a diminuição do tabaco, e ao ressaltar sempre a importância de se fazer o exame preventivo anualmente. ${ }^{7}$

É fundamental que os profissionais de saúde, dentre estes, os enfermeiros, tenham um 
olhar voltado para a detecção precoce do câncer do colo do útero, visto que a mortalidade por tal afecção pode estar relacionada às ações e políticas de prevenção deficitárias. ${ }^{8}$ Portanto, por entender a importância da rede de Atenção Primária à Saúde para prevenção e promoção da saúde, bem como para diagnosticar riscos e agravos, este estudo objetivou avaliar o seguimento clínico e terapêutico das coletas citopatológicas oncóticas de colo de útero nas Unidades Básicas de Saúde da Cidade de Santa Cruz/RN.

\section{Metodologia}

Trata-se de um estudo exploratóriodescritivo, de abordagem quantitativa e caráter avaliativo. Fizeram parte da amostra 150 mulheres entre 20 e 49 anos, residentes na Cidade de Santa Cruz/RN, Brasil, atendidas e cadastradas na rede APS, no período de julho a outubro de 2013.

O presente estudo é um recorte da pesquisa intitulada Cobertura de exames citopatológicos: perfil epidemiológico e avaliação do seguimento clínico e terapêutico nas mulheres. A coleta de dados foi realizada por meio de um formulário de entrevista desenvolvido pelos entrevistadores. As unidades de saúde e os sujeitos da pesquisa foram escolhidos mediante sorteio, contudo foram considerados como critérios de inclusão: ter mais de 20 anos; ser atendida na rede APS; possuir capacidade cognitiva para responder as perguntas e assinar o Termo de Consentimento Livre e Esclarecido (TCLE).

Os dados foram tabulados e analisados no Statistical Package for the Social Sciences (SPSS), versão 17.0. Para análise dos dados, foram calculadas as medidas de estatística descritiva como média, mediana e desvio-padrão e, no que diz respeito à análise bivariada, foi aplicado o teste do qui-quadrado, convencionando-se o intervalo de confiança de 95\% e como nível de significância $p<0,05$.
O estudo seguiu as recomendações constantes da Resolução nº 466/2012 do Conselho Nacional de Saúde e com a Declaração de Helsinki, referentes à pesquisa com seres humanos. O projeto de pesquisa foi apreciado pelo Comitê de Ética em Pesquisa (CEP) da Faculdade de Ciências da Saúde do Trairi-UFRN, para fins de avaliação e posterior aprovação, sendo aprovado sob parecer de $n^{\circ} 313.430$.

\section{Resultados}

Este estudo buscou avaliar o seguimento clínico e terapêutico das coletas citopatológicas oncóticas de colo de útero. Para isso, foi composta uma amostra de 150 mulheres, com idade mínima de 20 anos e máxima de 49, com média de 33,4 anos; mediana de 32,0 e desvio padrão de 7,6. No que diz respeito à escolaridade das entrevistadas, obteve-se uma média de 6,4 anos de estudo; mediana 6,0 e desvio padrão de 3,5. Em relação à renda familiar, a média foi de 495,2 reais; mediana de 400,0 e desvio padrão de 594,2 reais. Ainda sobre os dados sociodemográficos, $61,3 \%$ ( $n=92)$ moram em casa própria, $57,3 \%(n=86)$ são casadas e $22,7 \%(n=34)$ possuem união estável. Utilizou-se como critério de inclusão ter realizado o exame citopatológico alguma vez, e a média de anos entre um exame citopatológico e outro foram de três anos conforme preconizado, bem como terem apresentado o resultado do exame a algum profissional de saúde. Em relação ao tempo de espera para recebimento do exame, $52,0 \%(n=78)$ afirmaram ter demorado.

A tabela 1 mostra os dados referentes aos resultados das coletas de exames citológicos, a partir dos quais se observou que 66\% (n=99) tiveram resultados positivos para Cândida albicans, seguido por 28,7\% ( $\mathrm{n}=43$ ) que tiveram Gardnerella vaginallis. 
Quanto à ocorrência de familiares com história de câncer de colo do útero, $74,3 \%(n=113)$ das pessoas responderam não, seguido por $18 \%$ $(n=27)$ que tinham familiares com câncer de colo do útero. No que diz respeito a esse familiar ter sobrevivido ao câncer uterino, 14,0\% ( $n=21)$ das entrevistadas responderam que não e 4,0\% (n=06) afirmaram que sim. E, em relação ao parentesco dos familiares que apresentaram diagnóstico de câncer de colo do útero, $7,3 \%(n=11)$ das pessoas responderam tia, 3,3\% $(n=05)$ responderam mãe, $2,7 \%(n=04)$ responderam prima, 2,0\% ( $n=03)$ responderam irmã, 1,3\% $(n=02)$ responderam avó e 1,3\% $(n=02)$ responderam sobrinha. Quanto à idade em que esses familiares tiveram câncer do colo do útero, a média de idade foi de 45,1 anos.

A tabela 2 faz referência à participação das usuárias nas palestras de prevenção e detecção do câncer de colo do útero e, nesse quesito, 48,0\% $(n=72)$ das pessoas responderam que já ouviram ou já participaram de alguma palestra, seguido por $52 \%(n=78)$ que verbalizaram não ter participado. Quando questionadas sobre sua ida ao hospital de referência para realizar o tratamento, apenas $4,7 \%(n=07)$ das entrevistadas responderam que sim.

A tabela 3 é referente ao sentimento da mulher ao saber o resultado do exame citopatológico e aponta que $70,7 \% \quad(n=106)$ responderam que não se preocuparam com o resultado, enquanto $13,3 \%(n=20)$ responderam sentir ansiedade e 11,3\% ( $n=17)$ afirmaram ter sentido medo. Verificou-se ainda a correlação significativa com os resultados dos exames preventivo, comprovado pelo valor de $p<0,05$.

Quando questionadas sobre o atendimento que tiveram tanto na unidade de saúde quanto no hospital de referência, 4,7\% $(n=07)$ das

Tabela 1. Dados dos resultados dos exames preventivos. Santa Cruz, Rio Grande do Norte, Brasil, 2014.

\begin{tabular}{cccccc}
\hline & $\begin{array}{c}\text { Cândida } \\
\text { albicans }\end{array}$ & $\begin{array}{c}\text { Gardnerella } \\
\text { vaginallis }\end{array}$ & $\begin{array}{c}\text { HPV e NIC } \\
\text { baixo grau }\end{array}$ & $\begin{array}{c}\text { Trichomonas } \\
\text { vaginallis }\end{array}$ & Total \\
\hline Agravos & $66,0 \%$ & $28,7 \%$ & $4,7 \%$ & $0,7 \%$ & $100,0 \%$ \\
& $(n=99)$ & $(n=43)$ & $(n=07)$ & $(n=01)$ & $(n=150)$ \\
\hline
\end{tabular}

Tabela 2. Dados referentes à participação e detecção do câncer do colo do útero e a ida das mulheres ao hospital de referência. Santa Cruz, Rio Grande do Norte, Brasil, 2014.

\begin{tabular}{l|cc}
\hline & Sim & Não \\
\hline Participação em palestras sobre prevenção e detecção de câncer do colo & $48,0 \%$ & $52,0 \%$ \\
do útero & $(\mathrm{n}=72)$ & $(\mathrm{n}=78)$ \\
$4,7 \%$ & - \\
Ida ao hospital de referência & $(\mathrm{n}=07)$ &
\end{tabular}

Tabela 3 - Correlação entre os resultados do exame preventivo e o sentimento das mulheres ao receber o resultado. Santa Cruz, Rio Grande do Norte, Brasil, 2014.

\begin{tabular}{c|c|c|c|c|c|c|}
\hline \multicolumn{2}{|c|}{ Sentimento ao receber o resultado do exame } \\
$\begin{array}{c}\text { Não se } \\
\text { preocupou }\end{array}$ & Ansiedade & Medo & $\begin{array}{c}\text { Preocupação } \\
\text { com os filhos }\end{array}$ & Tristeza & $\begin{array}{c}\text { Preocupação } \\
\text { com a família }\end{array}$ & $p<0,05$ \\
\hline $\begin{array}{c}70,7 \% \\
(n=106)\end{array}$ & $\begin{array}{c}13,3 \% \\
(n=20)\end{array}$ & $\begin{array}{c}11,3 \% \\
(n=17)\end{array}$ & $\begin{array}{c}2,7 \% \\
(n=04)\end{array}$ & $\begin{array}{c}1,3 \% \\
(n=02)\end{array}$ & $\begin{array}{c}0,7 \% \\
(n=01)\end{array}$ & \\
\hline
\end{tabular}


entrevistadas responderam que fizeram exames, valor equivalente às mulheres que tiveram resultados de exames preventivos positivos de HPV e NIC I, enquanto 95,3\% ( $n=143$ ) responderam que tiveram outro tipo de atendimento. A saber: $58,7 \%(n=88)$ das mulheres responderam que - profissional de saúde prescreveu nistatina, seguido por $27,3 \%(n=40)$ às quais o profissional de saúde prescreveu metronidazol. As mulheres que tiveram resultados positivos para HPV e NIC não realizaram nenhum tipo de cirurgia e nem fizeram uso de quimioterapia ou radioterapia.

Ao perguntar às mulheres que tiveram resultado de exame preventivo positivo para HPV e NIC I se haviam retornado à unidade de saúde para realizar novamente o exame citopatológico após o tratamento, 3,3\% $(n=05)$ afirmaram sim, seguido por $1,3 \%$ ( $n=02)$ que verbalizaram não.

Quanto à classificação do atendimento realizado na Unidade Básica de Saúde e no hospital de referência, na ótica dos usuários, 53,3\% $(n=80)$ o classificou como bom, enquanto $38,7 \%$ $(n=58)$ o classificam como regular.

A tabela 4 faz referência às orientações feitas por profissionais de saúde. No que concerne às formas de prevenção e detecção do câncer de colo do útero, 53,3\% $(n=80)$ das mulheres afirmaram nunca ter tido tais orientações, já 20,0\% $(n=30)$ apontaram que raramente tiveram estas orientações. Quanto aos profissionais orientálas sobre a importância de realizar o exame preventivo, $34,0 \%(n=51)$ das mulheres falaram que sempre recebem orientações, $27,3 \%(n=41)$ falaram que raramente recebem orientações, seguido por $25,3 \%(n=38)$ que relataram algumas vezes receberem.

Quando questionadas se foram orientadas pelos profissionais a realizar o tratamento em outro hospital, 94,7\% ( $n=142)$ das mulheres responderam que não, uma vez que se tratava daquelas cujo resultado foi negativo, seguido de $5,3 \%(n=08)$ que responderam sim. No tocante ao fato de o hospital ser de referência ao tratamento oncológico, 4,7\% $(n=7)$ das entrevistadas falaram que não.

No que diz respeito à conduta dos profissionais de saúde diante do resultado do exame preventivo - tabela $5,94 \%(n=141)$ das mulheres responderam que os profissionais realizaram orientações em saúde, seguido por $4,0 \%(n=06)$ que afirmaram que o profissional as encaminhou para um médico especializado. Verificou-se ainda uma correlação significativa entre as variáveis: resultados dos exames preventivos e a conduta profissional, comprovado pelo valor de $p<0,05$.

Quanto às orientações dadas, $85,3 \%$ $(n=128)$ apontaram ter sido orientada quanto ao uso de pomada específica, 7,3\% ( $n=11)$ não receberam nenhum tipo de orientações, $4,7 \%$ $(n=07)$ foi orientada quanto ao tratamento do HPV e NIC I, 2,0\% ( $n=03)$ não se lembram das orientações recebidas e $0,7 \%(n=01)$ receberam orientações sobre a importância de realizar o exame preventivo.

Tabela 4- Dados referentes às orientações feitas pelos profissionais de saúde sobre o exame preventivo. Santa Cruz, Rio Grande do Norte, Brasil, 2014.

\begin{tabular}{c|c|c|c|c|c}
\hline \multicolumn{2}{c}{ Nunca } & Raramente & $\begin{array}{c}\text { Algumas } \\
\text { vezes }\end{array}$ & Frequentemente & Sempre \\
Prevenção e deteç̧ão & $53,3 \%$ & $20,0 \%$ & $16,7 \%$ & $2,0 \%$ & $8,0 \%$ \\
do câncer uterino. & $(\mathrm{n}=80)$ & $(\mathrm{n}=30)$ & $(\mathrm{n}=25)$ & $(\mathrm{n}=03)$ & $(\mathrm{n}=12)$ \\
Importância de fazer o & $12,7 \%$ & $27,3 \%$ & $25,3 \%$ & $0,7 \%$ & $34,0 \%$ \\
exame preventivo. & $(\mathrm{n}=19)$ & $(\mathrm{n}=41)$ & $(\mathrm{n}=38)$ & $(\mathrm{n}=01)$ & $(\mathrm{n}=51)$ \\
\hline
\end{tabular}


Tabela 5- Correlação entre os resultados dos exames preventivos e a conduta profissional. Santa Cruz, Rio Grande do Norte, Brasil, 2014.

\begin{tabular}{|c|c|c|c|c|c|c|c|}
\hline & & \multicolumn{5}{|c|}{ Conduta do profissional } & \multirow[b]{2}{*}{$p<0,05$} \\
\hline & & $\begin{array}{l}\text { Realizou } \\
\text { orientações } \\
\text { em Saúde } \\
\end{array}$ & $\begin{array}{c}\text { Encaminhou } \\
\text { para o } \\
\text { médico da } \\
\text { UBS }\end{array}$ & $\begin{array}{l}\text { Encaminhou } \\
\text { para o médico } \\
\text { especializado }\end{array}$ & $\begin{array}{c}\text { Não } \\
\text { orientou }\end{array}$ & Total & \\
\hline \multirow{4}{*}{$\begin{array}{l}\text { Resultados } \\
\text { do exame } \\
\text { preventivo. }\end{array}$} & NIC I e HPV & $\begin{array}{c}0,7 \% \\
(n=01)\end{array}$ & $\begin{array}{c}0,7 \% \\
(n=01)\end{array}$ & $\begin{array}{c}3,3 \% \\
(n=05)\end{array}$ & $\begin{array}{c}0,0 \% \\
(n=00)\end{array}$ & $\begin{array}{c}4,7 \% \\
(n=07)\end{array}$ & \multirow{5}{*}{$p<0,000$} \\
\hline & Cândida & $\begin{array}{l}64,0 \% \\
(n=96)\end{array}$ & $\begin{array}{c}0,0 \% \\
(n=00)\end{array}$ & $\begin{array}{c}0,7 \% \\
(n=01)\end{array}$ & $\begin{array}{c}1,3 \% \\
(n=02)\end{array}$ & $\begin{array}{l}66,0 \% \\
(n=99)\end{array}$ & \\
\hline & Gardnerella & $\begin{array}{l}28,7 \% \\
(n=43)\end{array}$ & $\begin{array}{c}0,0 \% \\
(n=00)\end{array}$ & $\begin{array}{c}0,0 \% \\
(n=00)\end{array}$ & $\begin{array}{c}0,0 \% \\
(n=00)\end{array}$ & $\begin{array}{l}28,7 \% \\
(n=43)\end{array}$ & \\
\hline & Trichomonas & $\begin{array}{c}0,7 \% \\
(n=01)\end{array}$ & $\begin{array}{c}0,0 \% \\
(n=00)\end{array}$ & $\begin{array}{c}0,0 \% \\
(n=00)\end{array}$ & $\begin{array}{c}0,0 \% \\
(n=00)\end{array}$ & $\begin{array}{c}0,7 \% \\
(n=01)\end{array}$ & \\
\hline \multicolumn{2}{|c|}{ Total } & $\begin{array}{c}94,0 \% \\
(n=141)\end{array}$ & $\begin{array}{c}0,7 \% \\
(n=01)\end{array}$ & $\begin{array}{c}4,0 \% \\
(n=06)\end{array}$ & $\begin{array}{c}1,3 \% \\
(n=02)\end{array}$ & $\begin{array}{c}100 \% \\
(n=150)\end{array}$ & \\
\hline & & & & & & & \\
\hline
\end{tabular}

\section{Discussão}

No que concerne aos dados sociodemográficos, observou-se que a população da pesquisa foi composta por mulheres jovens, as quais têm ensino fundamental incompleto e renda mínima inferior a um salário mínimo.

Os fatores sociodemográficos influenciam de forma bastante considerável para o surgimento e complicações do câncer de colo do útero. Considera-se que, quanto mais tardia a idade do diagnóstico, menores são as chances de cura nos casos das lesões precursoras do câncer uterino. Além disso, o fator escolaridade também é essencial, tendo em vista que quanto maior for o número de mulheres com o nível de ensino fundamental incompleto, de certa forma, maior poderá ser o número destas com diagnóstico tardio, grau (III e IV) da doença, justamente pela falta de busca de informações e, por conseguinte, o que pode contribuir para as recidivas, metástases e os casos de óbitos. ${ }^{9}$

Pode-se verificar ainda, que o fator estado civil pode influenciar a contaminação com o HPV e o diagnóstico de câncer de colo do útero. Uma vez que as mulheres casadas e com união estável são mais susceptíveis à contaminação por HPV, consequentemente, têm maior probabilidade de adquirir câncer. Por outro lado, as solteiras tendem a se prevenir mais, por não conhecerem bem seu parceiro.

No tocante aos resultados dos exames preventivos (tabela 1), constatou-se que a maioria das entrevistadas teve resultados positivos para Cândida albicans, seguido de Gardnerella vaginallis, tendo apenas sete resultados positivos para HPV e NIC I, e resultado inferior para Trichomonas vaginallis. De acordo com a literatura estudada, além da principal função de identificar as lesões iniciais do câncer uterino, o exame 
citopatológico pode detectar doenças sexualmente transmissíveis, como o HPV e as vulvovaginites (Trichomonas vaginallis, Cândida albicans e Gardnerella vaginalis).

Constatou-se a existência da necessidade urgente de programas com uma boa triagem, para que se possa rastrear o câncer uterino, com técnicas de rastreamento citológico e a detecção de HPV-DNA inclusas, entre outras técnicas, para melhor compreender como se dá a evolução do HPV em diferentes contextos epidemiológicos. ${ }^{10}$ A candidíase foi percebida como uma das mais presentes nos resultados de exames citológicos da pesquisa e mostrou, assim, que as mulheres estão cada vez mais suscetíveis a desenvolver essas infecções vaginais. Identificou-se que aquelas que apresentaram NIC em seus resultados de exames já tiveram antes e depois do NIC a Cândida albicans ou a Gardnerella vaginallis.

Referente aos familiares que tiveram câncer do colo do útero, percebe-se que a maioria foi a óbito por essa patologia e, quanto à faixa etária das mulheres que tiveram câncer de colo do útero, a média é de 45,1 anos de idade. Ressalta-se, assim, a importância de medidas de conscientização, educação em saúde, melhorias no atendimento à saúde da mulher, como meio para se ter um diagnóstico precoce da doença e um prognóstico que seja bom e, por consequência, haja uma diminuição na taxa de mortalidade.

De acordo com o Ministério da Saúde, a faixa etária de rastreamento do câncer do colo do útero é de 25 a 60 anos de idade, levando em consideração a história natural do câncer do colo do útero, que permite a detecção precoce das lesões pré-malignas ou malignas e o seu tratamento oportuno, graças à lenta progressão que este apresenta para a doença mais grave. ${ }^{11}$ Assim, a atuação na prevenção primária do câncer de colo do útero se dá nas medidas preventivas, evitando o acometimento da doença. As ações de prevenção primária acontecem de duas formas: a inespecífica, como a luta contra o tabagismo, educação sexual e uso de pílulas anticoncepcionais, e as especificas, como a vacinação e o exame Papanicolau. ${ }^{12}$

Em um estudo realizado ${ }^{13}$ constatou-se que existe desconhecimento, por parte das mulheres, em relação à forma de prevenção e deteç̧ão do câncer de colo do útero. Fato que mostra que as mulheres parecem não ter conhecimento sobre a finalidade do exame citopatológico. Nesse contexto, o exame seria para diagnosticar doenças já existentes e sintomáticas, ou outras doenças no corpo, sem relatar que o exame preventivo é a forma de rastrear e diagnosticar o câncer de colo do útero. O estudo evidencia ainda que a falta de conhecimento das mulheres é consequência de falhas no processo educativo, o qual deve ser realizado principalmente pela atenção básica, que deve priorizar a promoção da saúde, antes mesmo da detecção e tratamento dos agravos.

No que se refere à ida das mulheres ao hospital de referência, constatou-se que todas as mulheres que apresentaram HPV e NIC I foram ao hospital referenciado pelos profissionais da unidade básica. Os encaminhamentos foram feitos pelos enfermeiros, durante a consulta em que elas mostraram o resultado do exame preventivo, ou pelos médicos da UBS.

Percebeu-se que existe uma divergência na busca pelo hospital de referência entre as mulheres que apresentam as lesões precursoras e aquelas que já têm um alto grau de NIC e, até mesmo, o câncer uterino. ${ }^{14}$ Então, significa dizer que a busca pelo hospital de referência daquelas que tem NIC I se dá através de encaminhamentos, 
porém aquelas que já estão em um grau mais avançado tendem a procurar o serviço por conta própria, por já apresentarem sintomas.

Quanto aos sentimentos das mulheres ao receber o resultado do exame preventivo (tabela 4), a maioria declarou não se preocupar com o resultado, logo a ansiedade e o medo se mostraram menos presentes.

O sentimento mais presente nas mulheres ao receber o resultado do exame preventivo é a ansiedade, uma vez que é importante considerar que já fazem o exame preventivo repletas de medo, já que no momento da coleta, há o desconforto do próprio exame em si. Assim, é papel do profissional, ao realizar o procedimento cabível, explicar como se faz a coleta e tirar quaisquer dúvidas que as mulheres venham a apresentar. ${ }^{15}$

No que concerne ao tratamento realizado, as mulheres que apresentaram vulvovaginites referiram utilizar mais a nistatina e o fluconazol, já as que apresentaram HPV e NIC I, referiram a cauterização como forma mais frequente de tratamento. Quanto ao tratamento observou-se ${ }^{16}$ que, existem vários meios, entretanto os mais utilizados são azólicos, seja na forma oral, sejam tópicos, como o itraconazol e fluconazol, sendo, então, bastante eficazes.

Os resultados mostraram que a maioria das entrevistadas fez tratamento com nistatina. Uma observação interessante é que as vulvovaginites são sempre reincidentes. Fato que, muitas vezes, mostra como o tratamento acontece de forma inadequada. Desta forma, sobre o uso da nistatina, a mulher deve utilizá-la durante 14 dias e deve evitar ter relação sexual durante o período do tratamento. No entanto, geralmente não se segue as orientações dos profissionais.

Com relação à satisfação das usuárias quanto ao atendimento recebido, constatou-se que as usuárias consideram o atendimento recebido entre bom e regular. Justifica-se, então, pela prevalência de ótimo acolhimento, mesmo não existindo tanta ênfase para a promoção. Pois, como se percebeu nas entrevistas, as mulheres relataram não haver promoção à saúde.

Constatou-se que a avaliação positiva por parte das mulheres em relação ao atendimento prestado pelos profissionais de saúde durante o exame citopatológico é devida à competência técnica dos profissionais responsáveis pela coleta. ${ }^{17}$ Desta maneira, é evidente a importância da atuação dos profissionais na educação em saúde, para que promova a informação, o conhecimento em prol da saúde feminina, ou seja, como ocorre o exame preventivo, como realmente é a coleta, de maneira que transmita segurança às mulheres.

A promoção à saúde é de fundamental importância tanto para prevenir o câncer de colo do útero como qualquer outra doença, uma vez que é fator indispensável para proporcionar à população uma melhor condição de saúde através do conhecimento adquirido.

Sabe-se a importância da educação em saúde, principalmente, a utilização da sala de espera, quando as mulheres estão aguardando a realização do exame preventivo. Assim, o profissional de saúde deve aproveitar para thes explicar sobre o que aguardam, de maneira que ainda as motive a conhecer-se, tendo em vista que muitas delas não conhecem o próprio corpo, não têm nenhuma compreensão do exame preventivo, nem do câncer de colo do útero. ${ }^{18}$

No tocante às condutas dos profissionais de saúde perante os resultados dos exames preventivos, tabela 5 , evidenciou-se que a maioria 
das mulheres recebe orientações em saúde, e aquelas que tiveram resultados positivos para HPV e NIC I relataram terem sido encaminhadas para um médico, seja ele um profissional especializado ou o médico da UBS. Quanto às orientações dadas pelos profissionais de saúde, a maioria das mulheres relatou ter recebido orientações quanto ao uso de creme vaginal (pomada), já as que tiveram resultados positivos receberam orientações sobre o tratamento do HPV e NIC I.

Assim, observa-se a necessidade de os profissionais utilizarem o diálogo como ferramenta de cuidado e prevenção durante a coleta citopatológica, bem como o retorno para mostrar o resultado do exame preventivo, de modo que busque esclarecer eventuais dúvidas das mulheres e favoreça um melhor acolhimento e satisfação das usuárias. ${ }^{19}$

Desta forma, tem-se o papel do profissional

\section{REFERÊNCIAS}

1. Greenwood AS, Machado MFAS, Sampaio NMV. Motivos que levam mulheres a não retornarem para receber o resultado de exame Papanicolau. Rev Latino-Am Enfermagem. 2006; 14(4):503-509.

2. Teixeira LA, Porto MAT, Souza LPA. A expansão do rastreio do câncer do colo do útero e a formação de citotécnicos no Brasil. PhysisRevista de Saúde Coletiva. 2012; 22(2):713-731.

3. BRASIL. Ministério da Saúde. Secretaria de Atenção à Saúde, Departamento de Ações Programáticas Estratégicas. Política Nacional de Atenção Integral à Saúde da Mulher Princípios e Diretrizes. Brasilia-DF, 2009.

4. INCA ( Instituto Nacional de Câncer). Ministério da Saúde. Incidência de câncer no Brasil. 2012. Disponível em: <http://www.inca.gov.br/estimativa/2012/ tabelaestados.asp? UF=BR>. Acesso em: 28 de Agosto de 2012.

5. Cruz LMB, Loureiro RP. A Comunicação na Abordagem Preventiva do Câncer do Colo do Útero: importância das influências histórico-culturais e da sexualidade feminina na adesão às campanhas. Saúde Soc. 2008; 17(2):120-31.

6. Soares $\mathrm{MC}$ et al. Câncer de colo uterino: atenção integral à mulher nos serviços de saúde. Rev Gaúcha Enferm. 2011; 32(3):502-508.

7. Guimarães JAF et al. Pesquisa brasileira sobre prevenção do câncer de colo uterino: uma revisão integrativa. Rev Rene. 2012;13(1):220-230. de saúde como fundamental para promover à assistência contínua a mulher, estimular práticas de prevenção e promoção à saúde desta e, assim, diminuir as taxas de mulheres com CCU e outras patologias evitáveis. Cabe destacar ainda a importância da APS como meio para prestar uma assistência qualificada e resolutiva às necessidades da mulher, por se articular com os demais níveis de assistência.

Espera-se que o referido estudo sensibilize gestores e profissionais de saúde, para que possam elaborar estratégias que venham a fortalecer o vínculo entre o usuário e o serviço e, assim, promover o acompanhamento das mulheres no serviço, possibilitando o conhecimento sobre o exame preventivo e as doenças susceptíveis, de maneira que estabeleçam uma relação de confiança e estimulem práticas de prevenção e autocuidado.

8. Thum $\mathrm{M}$ et al. Câncer de colo uterino: Percepção das mulheres sobre prevenção. CiencCuid Saúde. 2008; 7(4):509-516.

9. Mascarello KC et al. Perfil sociodemográfico e clínico de mulheres com câncer do colo do útero associado ao estadiamento inicial. Revista Brasileira de Cancerologia. 2012; 58(3):417-426.

10. Vieira L, Almeida A. The cytology and dna detection by the papillocheck ${ }^{\circledR}$ test in the diagnosis of human papillomavirus infection. European Journal of Microbiology and Immunology. 2013; 3(1):61-67.

11. BRASIL. Ministério da Saúde. Secretaria de Atenção à Saúde. Departamento de Atenção Básica. Controle dos cânceres do colo do útero e da mama. Cadernos de Atenção Básica n. 13. Série A. Normas e manuais técnicos. Ministério da Saúde, Brasília-DF, 2006.

12. Cestari MEW, Zago MMF. A prevenção do câncer e a promoção de saúde: um desafio para o Século XXI. Rev Bras Enferm. 2005; 58(2):218-221.

13. Garcia CL, Pereira HC, Marinho MNASB. Percepções das mulheres acerca do exame de prevenção do câncer cérvico-uterino. RBPS. 2010; 23(2):118-125.

14. Guarisi R et al. Rastreamento, Diagnóstico e Tratamento das Lesões Precursoras e do Câncer Invasor de Colo Uterino no Município de Franco da Rocha, SP. Revista Brasileira de Cancerologia. 2004; 50(1):7-15. 
15. Jorge RJB et al. Exame Papanicolau: sentimentos relatados por profissionais de enfermagem ao se submeterem a esse exame. Ciênc. Saúde Coletiva. 2011; 16(5):2443-2451.

16. Zimmermmann BL et al. Avaliação do tratamento antifúngico oral e tópico-oral para candidíase vulvovaginal. HU Revista. 2009; 35(3):167-173.

17. Oliveira WMA et al. Adesão de mulheres de 18 a 50 anos ao exame colpocitológico na estratégia saúde da família. Rev. Enf.2012; (7):15-22.

18. Rodrigues $\mathrm{BC}$ et al. Educação em saúde para a prevenção do câncer cérvico-uterino. Rev. Bras. Educ. Med. 2012. 36(suppl.1):149-154.
19. Andrade SSC et al. Compreensão de usuárias de uma Unidade de Saúde da Família sobre o exame Papanicolau. Ciênc. Saúde Coletiva. 2013; 18(8):2301-2310.

\section{CORRESPONDÊNCIA}

Tainara Lôrena dos Santos Ferreira

Faculdade de Ciências da Saúde do Trairi (FACISA) / UFRN Rua Trairi, S/N, Centro, Santa Cruz/RN; CEP: 59.200-000.

E-mail: tainara_lorena@hotmail.com 